\title{
Panax notoginseng (Burk.) Effects on Fibrinogen and Lipid Plasma Level in Rats Fed on a High-fat Diet
}

\author{
A. F. G. Cicero, ${ }^{1 *}$ G. Vitale, ${ }^{2}$ G. Savino ${ }^{2}$ and R. Arletti ${ }^{2}$ \\ ${ }^{1}$ Athensclensis Study Center, 'G Descovich', University of Bologna, Italy \\ ${ }^{2}$ Biomedical Sciences Department, Pharmacology Section, University of Modena and Reggio Emilia, Italy
}

\begin{abstract}
Several studies have shown that notoginsenoides improve diastolic function in hypertensive subjects, induce the fibrinolytic system in in vitro models and act as antiproliferative agents on vessel leiomyocytes. Our aim was to evaluate their effect on fibrinogen and lipid plasma levels compared with a well-known HMGCoA reductase inhibitor. Seventy Wistar male adult rats on a fat-enriched diet were treated orally with $P$. notoginseng pulverized root $(43 \mathrm{mg} / \mathrm{kg} / \mathrm{day}$ or $86 \mathrm{mg} / \mathrm{kg} / \mathrm{day} ; 20$ animals per group), fluvastatin $(3 \mathrm{mg} / \mathrm{kg} / \mathrm{day} ; 20$ animals) or physiological saline $(5 \mathrm{~mL} / \mathrm{kg} / \mathrm{day} ; 10$ animals). The ten rats on a normocaloric diet were also treated with $5 \mathrm{~mL} / \mathrm{kg} /$ day of physiological saline. After a 28-day treatment, the rats were killed and their blood analysed with standard procedures. Treatment with $43 \mathrm{mg} / \mathrm{kg} / \mathrm{day}$ of $P$. notoginseng or $3 \mathrm{mg} / \mathrm{kg} /$ day of fluvastatin showed similar activity in decreasing total cholesterol $(-23.70 \%$, $-19.29 \%$, respectively) and triglycerides $(-21.59 \%,-18.55 \%)$. The most evident effect of $P$. notoginseng was the reduction of fibrinogenaemia in treated rats compared with the control values $(-38.10 \%$; $p<0.001$, no dose-relationship being shown in this effect. Moreover, no significant variation in HDL cholesterol and glucose levels was observed nor did relevant behavioural changes occur in association with the root intake. Besides a moderate, non dose-related decrease in the plasma lipid levels, $P$. notoginseng appeared to induce a significant reduction in the rat fibrinogenaemia. Copyright (C) 2003 John Wiley \& Sons, Ltd.
\end{abstract}

Keywords: cholesterol; fibrinogen; Panax notoginseng; herbal remedies.

\section{INTRODUCTION}

The potential use of Arialiaceae roots in cardiovascular disease prevention was first recognized by Japanese authors 20 years ago. In fact, Panax ginseng (Renshen) administration induced a decrease in low density lipoprotein cholesterol (LDL-C) triglycerides (TG) plasma and liver levels, as well as an increase in the high density lipoprotein cholesterol (HDL-C) plasma levels and a reduction of platelet adhesiveness in both rats (Yamamoto et al., 1983a) and hyperlipidaemic patients (Yamamoto et al., 1983b; Punnonen and Lukola, 1984). The proposed mechanisms of action were the following: an increase in faecal fat excretion, a decrease in the activities of $\beta$-hydroxy- $\beta$-methylglutaryl-CoA reductase, acyl-coenzyme-A-cholesterol acyltransferase and cholesteryl ester transfer protein (Qureshi et al., 1983; Yamamoto et al., 1983b; Punnonen and Lukda, 1984; Kwon et al., 1996; Kwon et al., 1999).

Panax notoginseng (Burk.) F. H. Chen (Sanqi) is a root, similar to the more well-known and studied $P$. ginseng (Ngan et al., 1999). All the Arialiaceae of the ginseng group have a similar composition, even if the chemical constituents have a different concentration, specificity and activity (Bruneton, 1999). Recent studies

\footnotetext{
* Correspondence to: A. F. G. Cicero, Athensclensis Study Center, 'G Descovich', Clinical Medicine and Applied Biotechnology Department, D. Campanacci, Via Massarenti 9, 40139 Bologna, Italy.

E-mail: afgcicero@tiscalinet.it
}

demonstrated various specific pharmacological actions of notoginsenoides, especially on the cardiovascular system. The chronic administration of $P$. notoginseng with the ACE-inhibitor captopril improved the left ventricular diastolic function in hypertensive patients compared with treatment with captopril alone; this effect was partially related to an antagonism to selective $\mathrm{Ca}^{++}$receptor operating channels (Feng et al., 1999). Among the haemorheological parameters, notoginsenoides appeared to increase the synthesis of the tissue-type plasminogen activator and to decrease the activity of plasminogen activator inhibitor-1 in cultured human endothelial and vascular smooth muscle cells from different vascular regions (Zhang et al., 1997). Moreover, a 10-day oral administration of $P$. notoginseng increased prostacyclin synthesis in the carotid artery and decreased thromboxane $\mathrm{A}_{2}$ synthesis in blood platelets; at the end of the experiment, aortic atherosclerotic plaque development was reduced (Shi et al., 1990). In a previous study we evaluated the adaptogenic properties of the root (Cicero et al., 2000). The aim of this study was to evaluate the effects of orally administered $P$. notoginseng $(43 \mathrm{mg} / \mathrm{kg}$ and $86 \mathrm{mg} / \mathrm{kg}$ in a volume of $5 \mathrm{~mL} / \mathrm{kg}$ ) on plasma lipid levels and fibrinogenaemia in rats on a high-fat diet.

\section{MATERIALS AND METHODS}

Eighty adult male Wistar rats (Harlan Italy, Correzzana, 
Table 1. Components and chemical analysis of fat diet

\begin{tabular}{lrlr}
\hline Component & $\mathrm{g} / \mathrm{kg}$ & Chemical analysis & $\%$ \\
\hline Casein & 220 & Water & 12.0 \\
Maize starch & 256 & & \\
Saccharose & 100 & & 19.0 \\
Cellulose & 40 & Raw proteins & \\
Bacon & 100 & Raw lipids & 4.0 \\
Soybean oil & 70 & As-spun & 7.0 \\
Coconut oil $_{\text {Salt-mineral mixture }}^{\mathrm{a}}$ & 130 & Raw ash & 44.5 \\
Oligoelements $^{\mathrm{a}}$ & 52 & Nitrogen-free extract & \\
Vitamin mixture $^{\text {DL-Methionine }}$ & 8 & & 100.0 \\
Total & 20 & & \\
\hline
\end{tabular}

${ }^{\text {a }}$ Salt-mineral mixture and vitaminized starch were made according to Seetharamaiah et al. [23].

Milan, Italy) weighing $240 \pm 20 \mathrm{~g}$ (3 months old on arrival) were used and housed 1 per cage in a temperature- $\left(22^{\circ} \pm 1 \mathrm{C}^{\circ}\right)$ and humidity- $(60 \%)$ controlled environment. The light/dark cycle was the natural one (the light phase of the cycle beginning at 6 a.m.). The animals were divided into two groups: ten rats (NC: normal diet controls) had free access to standard rat food in pellets (MIL, Morini, S. Polo D’Enza, Reggio nell'Emilia, Italy), 70 animals were fed with a high-fat diet containing $19 \%$ proteins and $25.5 \%$ lipids $(10 \%$ cholesterol). Water was available ad libitum. This dietary regimen was adopted throughout the experiment. Housing conditions and experiments were in accordance with the European Community regulations on the care and use of animals for scientific purposes (CEE Council 86/609, included in the Italian writ DL 27/01/92 N. 116). The rats were acclimatized to our housing conditions for at least 1 week before being used.

Drugs and treatment. Rats were orally treated through a gastric tube for 28 days consecutively. The rats on the fat diet were divided into four subgroups: ten were treated with $5 \mathrm{~mL} / \mathrm{kg} /$ day of saline (FC: fat diet controls), 20 were given $3 \mathrm{mg} / \mathrm{kg} /$ day of fluvastatin (FL: fluvastatin), 20 received $43 \mathrm{mg} / \mathrm{kg} /$ day of high quality $P$. notoginseng pulverized root (Ng) (Yunnan Medicines \& Health Products Corp., Yunnan, R. P. China) and 20: $86 \mathrm{mg} / \mathrm{kg} /$ day of $P$. notoginseng (NG). The NC animals were also treated with $5 \mathrm{~mL} / \mathrm{kg} /$ day of saline solution. At the end of the experiment, the animals were fasted overnight and killed under ether anaesthesia. Blood was collected by heart puncture. Plasma samples were stored at $-20^{\circ} \mathrm{C}$ before being used for analysis.

Laboratory analysis. Lipid levels [total cholesterol (TC), triglycerides (TG) and high density lipoprotein cholesterol (HDL-C)] were analysed on plasma samples by enzymatic-colorimetric methods according to standard laboratory techniques. The concentration of LDL cholesterol (LDL-C) was estimated through the Friedewald formula: LDL-C $=\mathrm{TC}-(\mathrm{TG} / 5+\mathrm{HDL}-\mathrm{C})$ for $\mathrm{TG}$ value $<400 \mathrm{mg} / \mathrm{dL}$. Glycaemia was also determined by the enzymatic-colorimetric method, as described by Gaddi et al. (2001). Fibrinogen levels were measured in duplicate using the Clauss method (Data-Fi Fibrinogen determination Kit, Dade County, FL) (Clauss, 1957).
Microstructural analysis of unconditioned behaviour. The test was carried out to detect any possible $P$. notoginseng-induced behavioural changes. Thirty minutes after the treatment, the observation started by keeping the animals in their individual home cages. The following parameters were separately registered on a specific schedule of $1 \mathrm{~h}$ periods: number and duration time dedicated to feeding, drinking, exploring, resting and grooming. Pre-weighed food pellets were provided at the beginning of the test and food intake was measured at the end by weighing the remaining food plus spillage. During the experiment the rats had free access to water.

Statistical analysis. Data were statistically processed by the Shapiro-Wilk normality test and analysis of variance (ANOVA-test) followed by Student's one-tail $t$-test (SPSS 8.0, Windows version, statistical software). A $p=0.05$ significance level was chosen for all statistical tests (Normann and Streiner, 2000).

\section{RESULTS}

Before killing the rats' mean weight had increased by $20 \%(301 \pm 19 \mathrm{~g})$ with no differences between the NC and FC animals. This fat diet model produced a significant increase in TC $(F=77.59, p<0.001)$, LDL$\mathrm{C}(F=64.76, p<0.001)$ and TG $(F=185.12, p<0.001)$ plasma levels, with no influence on HDL-C, glucose and fibrinogen levels $(p>0.05)$ compared with the $\mathrm{NC}$ values. Treatment with fluvastatin significantly lowered TC $(F=39.94, p<0.001)$, LDL-C $(F=9.98, p=0.005)$ and TG $(F=17.99, p<0.001)$ levels compared with those of the FC group, as expected by the literature data (Langtry and Markham, 1999). No relevant differences were observed in the TC $(F=4.31, p=0.053)$, LDL-C $(F=4.25, p=0.054)$ and TG $(F=0.76, p<0.428)$ levels between fluvastatin treated rats on a fat diet and controls on a standard diet. The administration of fluvastatin did not reduce fibrinogenaemia in comparison with $\mathrm{NC}$ and FC rats.

$43 \mathrm{mg} / \mathrm{kg} /$ day of $P$. notoginseng pulverized root significantly decreased TC $(F=20.84, \quad p<0.001)$, LDL-C $\quad(F=19.42, \quad p<0.001)$ and TG $(F=9.58$, $p<0.006)$ levels compared with the FC group. No relevant differences between the lower dose P. notogin- 


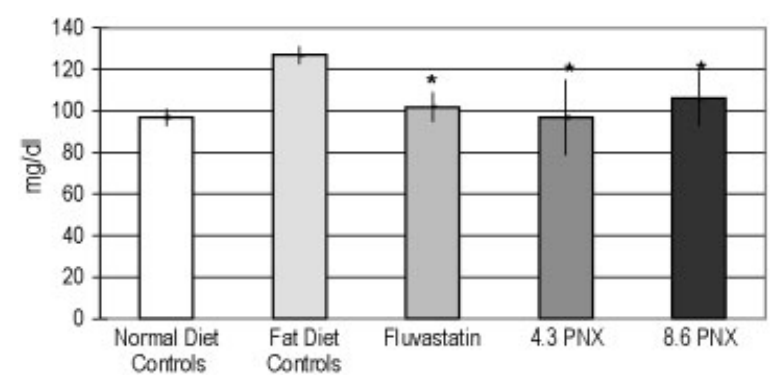

Figure 1. Effect of fat diet, fluvastatin $3 \mathrm{mg} / \mathrm{kg}$ and $P$. notoginseng (PNX) pulverized root $(43$ and $86 \mathrm{mg} / \mathrm{kg}$ ) on rat plasma total cholesterol level. ${ }^{*} p<0.01$ versus FC.

seng treated rats on a fat diet and controls on a standard diet were observed for TC $(F=0.00, p=0.987)$, LDL-C $(F=0.00, p=0.975)$ and TG $(F=0.01, p<0.936)$ levels. The $\mathrm{Ng}$ treatment reduced fibrinogenaemia compared with NC $(F=36.60, p<0.001)$ and FC $(F=51.95$, $p<0.001)$ rats.

$86 \mathrm{mg} / \mathrm{kg} /$ day of $P$. notoginseng pulverized root significantly lowered TC $(F=15.66, p<0.001)$, LDL$\mathrm{C}(F=11.93, p=0.003)$ and TG $(F=6.14, p<0.023)$ levels compared with the FC group. No relevant differences between the higher dose $P$. notoginseng treated rats on a fat diet and controls on a standard diet were observed concerning TC $(F=4.16, p=0.056)$, LDL-C $\quad(F=0.00, \quad p=1.000)$ and TG $(F=4.40$, $p=0.051)$ levels. The NG treatment decreased fibrinogenaemia compared with $\mathrm{NC}(F=20.12, p<0.001)$ and FC $(F=57.51, p<0.001)$ rats.

No significant difference was found between fluvastatin and $\mathrm{Ng}$ treated groups in TC $(F=0.81, p=0.381)$, LDL-C $\quad(F=2.46, \quad p=0.134) \quad$ and $\mathrm{TG} \quad(F=0.14$, $p=0.713)$. Unlike fluvastatin, $\mathrm{Ng}$ significantly lowered the fibrinogen level. Similar results were found comparing fluvastatin and $\mathrm{NG}$ treated rats. $\mathrm{Ng}$ and $\mathrm{NG}$ showed similar activity in reducing TC $(F=1.64, p=0.217)$, LDL-C $\quad(F=3.37, \quad p=0.083) \quad$ and TG $(F=1.34$, $p=0.263)$, while $\mathrm{Ng}$ seemed to be more effective in reducing the fibrinogen level than NG $(F=15.74$, $p<0.001)$.

Changes in TC, LDL-C, TG and fibrinogen plasma levels are reported in Figs 12 2 $3-4$. Microstructural analysis of the spontaneous behaviour revealed that both doses of orally administered $P$. notoginseng significantly increased the exploring time $(p<0.03)$ while reducing the grooming $(p<0.001)$ and resting times $(p<0.03)$. Once again, both $P$. notoginseng doses produced a greater number of exploring episodes $(p<0.01)$, and a lower number of grooming $(p<0.03)$ and resting episodes $(p<0.05)$ than the control rats. No significant differences in the duration and number of the episodes were observed between the $43 \mathrm{mg} / \mathrm{kg}$ and the $86 \mathrm{mg} / \mathrm{kg} P$. notoginseng-treated rats $(p>0.05)$. Finally, no significant difference was found in either feeding or drinking behaviour $(p>0.05)$.

\section{DISCUSSION}

The utility of some natural products in the treatment and prevention of atherosclerosis-related cardiovascular disease has been rigorously tested on animal and human



Figure 2. Effect of fat diet, fluvastatin $3 \mathrm{mg} / \mathrm{kg}$ and $P$. notoginseng (PNX) pulverized root $(43$ and $86 \mathrm{mg} / \mathrm{kg}$ ) on rat plasma low density lipoprotein level. ${ }^{*} p<0.01$ versus FC.

models. Currently the non-pharmaceutical aids in moderate dyslipidaemias are soy bean proteins for hypercholesterolaemias (Anderson et al., 1995), fish oil for hypertriglyceridaemias and hypoHDLaemias (GISSI, 1999), rice bran oil for moderate hypercholesterolaemias and hypertriglyceridaemias (Cicero and Gaddi, 2001). Recent studies show that high fibrinogen is an emerging independent risk factor for cardiovascular diseases (Harjai, 1999). In healthy non-smoking men, its level is associated with the expression of a more atherogenic LDL subfraction (small and dense LDL with $d>1.044$ $\mathrm{g} / \mathrm{dL}$ ), and a lower HDL-C level, in a way that is independent of body mass index, age and insulin resistance (Halle et al., 1996). In contrast, the only drugs that actually demonstrate a lowering effect on this parameter are the fibrates (bezafibrate, fenofibrate and ciprofibrate, but not gemfibrozil) that induced a fibrinogenaemia decrease of $-10 \%$ to $-30 \%$ in different clinical trials (Pazzucconi et al., 1992; Broijersen et al., 1996). In contrast to fibrates, $\beta$-hydroxy- $\beta$-methylglutaryl-CoA reductase inhibitors (statins) generally induce only a slight effect on the fibrinogen blood level (Nair et al., 1998; Jones et al., 1998). However, recent reports indicate an association between atorvastatin administration, a very potent lipid lowering agent, and fibrinogenaemia increase (ranging from $+20 \%$ to $+50 \%$ ) in some treated patients (Wierzbicki et al., 1998; Sinzinger and Rodrigues, 1999). The aim of our study was to confirm the decreasing effect of $P$. notoginseng on plasma lipids and to evaluate its effect on the fibrinogen plasma levels in rats fed a new model of a fat diet compared with the activity of a well-known effective statin (Langtry and Markham, 1999). We know that, theoretically, we had to

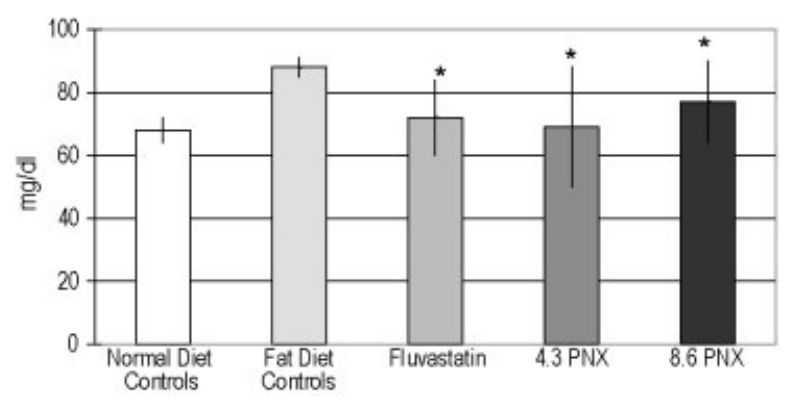

Figure 3. Effect of fat diet, fluvastatin $3 \mathrm{mg} / \mathrm{kg}$ and $P$. notoginseng (PNX) pulverized root $(43$ and $86 \mathrm{mg} / \mathrm{kg}$ ) on rat plasma triglyceride level. ${ }^{*} p<0.05$ versus FC. 


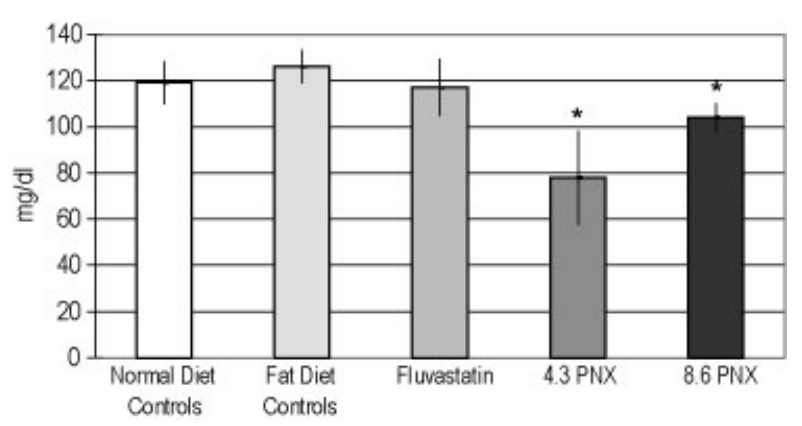

Figure 4. Effect of fat diet, fluvastatin $3 \mathrm{mg} / \mathrm{kg}$ and $P$. notoginseng (PNX) pulverized root $(4.3$ and $8.6 \mathrm{mg} / \mathrm{kg})$ on rat plasma fibrinogen level. ${ }^{*} p<0.01$ versus FC.

use a fibrate or pravastatin as a positive control to test the $P$. notoginseng efficacy to reduce the fibrinogenaemia, but we did not expect such a marked effect of the root on this parameter. The $P$. notoginseng doses were planned on the basis of the Traditional Chinese Medicine prescriptions for the treatment of angina pectoris and dyslipidaemias. The role of the root components (flavonoids, saponins, notoginsenoides and others) in cardiovascular disease management may be due to different properties: $\mathrm{Ca}^{++}$-channel blocking activity, improvement of diastolic function in hypertensive patients (Feng et al., 1999), increase in rTPA and decrease in PAI-1 synthesis in cultured human endothelium cells (Zhang et al., 1997) and correction of an inbalance between prostacyclin and thromboxane $\mathrm{A}_{2}$ (Shi et al., 1990). Our data confirm that orally administered $P$. notoginseng pulverized root produced a significant, non dose-related, lipid lowering effect compared with controls and a well-known effective treatment in hyperlipidaemic rats. $43 \mathrm{mg} / \mathrm{kg} /$ day of $P$. notoginseng and $3 \mathrm{mg} / \mathrm{kg} /$ day of fluvastatin showed a similar activity in decreasing plasma TC $(-23.70 \%$ and $-19.29 \%$, respectively) and TG (-21.59\% and $-18.55 \%)$ levels, while no significant change in HDL-C and glucose levels was observed $(p>0.05)$. The most important $P$. notoginseng effect was the mean reduction in fibrinogenaemia compared with the control values $(-38.10 \% ; F=51.95$, $p<0.001)$. No relevant behavioural modification was associated with the root intake. Just as all herbal remedies that consist of a mixture of many compounds, $P$. notoginseng root exerts its several pharmacological actions on different parameters. We suggest that further molecular studies are needed on the $P$. notoginseng root to identify the different components of this root in order to correlate molecular structure and effects. Since only a small fraction of the world is plant biodiversity has been tested for biological activity, it can be assumed that natural products will continue to offer new leads for innovative therapeutic agents.

\section{REFERENCES}

Anderson JW, Johnstone BM, Cook-Newell ME. 1995. Metaanalysis of the effects of soy protein intake on serum lipids. N Engl J Med 333: 276-282.

Broijersen A, Eirksson M, Wiman B, Angelin B, Hjemdahl P. 1996. Gemfibrozil treatment of combined hyperlipoproteinemia. No improvement of fibrinolysis despite marked reduction of plasma triglyceride levels. Arterioscler Thromb Vasc Biol 16: 511-516.

Bruneton J. 1999. Pharmacognosie. Phytochimie. Plantes Medicinales, 3rd edn. Edition Médicales Internationales: Cachan, France: 707-710.

Cicero AFG, Bandieri E, Arletti R. 2000. Panax notoginseng influences rat spontaneous behaviour. J Ethnopharmacol 73: 387-391.

Cicero AFG, Gaddi A. 2001. Rice bran oil and $\gamma$-oryzanol in the treatment of hyperlipoproteinemias and other conditions. Phytother Res 15: 277-289.

Clauss A. 1957. Quick method to estimate fibrinogen by a functional clotting assay. Acta Haematol 17: 237-246.

Feng PF, Oin NP, Qiao Q. 1999. Clinical and experimental study of improving left ventricular diastolic function by total saponins of Panax notoginseng. Chung Kuo Hsi I Chieh Ho Tsa Chih 17: 714-717.

Gaddi A, Cicero AFG, Dormi A, Pasquarelli V, D'Addato S. 2001. The realisation of a project aimed at reducing the plasmatic lipid level in a large Italian rural population improves the mean calcium daily intake: the Brisighella Study (BS). Eur J Clin Nutr 55: 97-106.

Gruppo Italiano per lo Studio della Sopravvivenza nell'Infarto Miocardico. 1999. Dietary supplementation with $\omega-3$ polyunsaturated fatty acids and vitamin E after myocardial infarction: results of the GISSI-Prevention trial. Lancet 354: 447-455.

Halle M, Berg A, Keul J, Baumstark MW. 1996. Association between serum fibrinogen concentrations and HDL and LDL subfraction phenotypes in healthy men. Arterioscler Thromb Vasc Biol 16: 144-148.

Harjai KJ. 1999. Potential new cardiovascular risk factors: left ventricular hypertrophy, homocysteine, lipoprotein(a), tryglicerides, oxidative stress and fibrinogen. Ann Intern Med 131: 376-386.

Jones $P$, Kafonek S, Laurora I, Hunninghake D, for the CURVES Investigators. 1998. Comparative dose efficacy study of atorvastatin versus simvastatin, pravastatin, lovastatin and fluvastatin in patients with hypercholesterolemia (The CURVES Study). Am J Cardiol 81: 582587.

Kwon BM, Kim MK, Baek NI et al. 1999. Acyl-CoA: cholesterol acyltransferase inhibitory activity of ginseng sapogenins, produced from the ginseng saponins. Bioorg Med Chem Lett 17: 1375-1378.

Kwon BM, Nam JY, Lee SH, Jeong TS, Kim YK, Bok SH. 1996. Isolation of cholesteryl ester protein inhibitors from Panax ginseng roots. Chem Pharm Bull 44: 444-445.

Langtry HD, Markham A. 1999. Fluvastatin. A review of its use in lipid disorders. Drugs 57: 583-606.

Nair DR, Papadakis JA, Jagroop IA, Mikhailidis DP, Winder AF. 1998. Statins and fibrinogen. Lancet 351: 1430-1431.

Ngan F, Shaw P, But P, Wang J. 1999. Molecular authentication of Panax species. Phytochemistry 50: 787-791.

Normann G, Streiner D. 2000. Biostatistics. The Bare Essentials, 2nd edn. B. C. Decker Inc.: Ontario, Canada: 89-162.

Pazzucconi F, Mannucci L, Mussoni L et al. 1992. Bezafibrate lowers plasma lipids, fibrinogen and platelet aggregability in hypertriglyceridemia. Eur J Clin Pharmacol 443: 219-223.

Punnonen R, Lukola A. 1984. The effect of ginseng on serum total cholesterol, HDL- cholesterol and triglycerides levels in postmenopausal women. Asia Oceania J Obstet Gynaecol 10: 399-401.

Qureshi AA, Abuirmeileh N, Din ZZ, Amhad Y, Burger WC, Elson CE. 1983. Suppression of cholesterogenesis and reduction of LDL cholesterol by dietary ginseng and its fractions in chicken liver. Atherosclerosis 48: 81-94.

Shi L, Fan PS, Wu L, Fang JX, Han ZX. 1990. Effects of total 
saponins of Panax notoginseng on increasing $\mathrm{Pgl}_{2}$ in carotid artery and decreasing $\mathrm{TxA}_{2}$ in blood platelets. Chung Kuo Yao Li Hsueh Pao 11: 29-32.

Sinzinger H, Rodrigues M. 1999. Atorvastatin and fibrinogen-a small subgroup shows extreme response. Atherosclerosis 145: 415-417.

Wierzbicki AS, Lumb PJ, Semra YK, Crook MA. 1998. Effect on atorvastatin on plasma fibrinogen. Lancet 351: 569570.

Yamamoto M, Kugamai A, Yamamura Y. 1983a. Plasma lipid- lowering action of ginseng saponins and mechanism of the action. Am J Chin Med 11: 84-87.

Yamamoto M, Uemura T, Nakama S, Uemiya M, Kumagai A. 1983b. Serum HDL-cholesterol- increasing and fatty liverimproving actions of Panax ginseng in high cholesterol diet-fed rats with clinical effect on hyperlipidemia in man. Am J Chin Med 11: 96-101.

Zhang WJ, Wojta J, Binder BR. 1997. Effect of notoginsenoides $R_{1}$ on the synthesis of components of the fibrinolytic system in cultured smooth muscle cells of human pulmonary artery. Cell Mol Biol 43: 581-587. 